\title{
Applications of proteomics to osteoarthritis, a musculoskeletal disease characterized by aging
}

\section{Ali Mobasheri *}

Musculoskeletal Research Group, Division of Veterinary Medicine, School of Veterinary Medicine and Science, University of Nottingham, Nottingham, UK

\section{Edited by:}

Alexandrina Ferreira Mendes,

University of Coimbra, Portugal

\section{Reviewed by:}

Alexandrina Ferreira Mendes, University of Coimbra, Portugal

\section{*Correspondence:}

Ali Mobasheri, School of Veterinary Medicine and Science, University of Nottingham, Sutton Bonington Campus, Sutton Bonington, Nottingham, Leicestershire LE12 5RD, UK.

e-mail:ali.mobasheri@nottingham. ac.uk
The incidence of age-related musculoskeletal impairment is steadily rising throughout the world. Musculoskeletal conditions are closely linked with aging and inflammation. They are leading causes of morbidity and disability in man and beast. Aging is a major contributor to musculoskeletal degeneration and the development of osteoarthritis (OA). OA is a degenerative disease that involves structural changes to joint tissues including synovial inflammation, catabolic destruction of articular cartilage and alterations in subchondral bone. Cartilage degradation and structural changes in subchondral bone result in the production of fragments of extracellular matrix molecules. Some of these biochemical markers or "biomarkers" can be detected in blood, serum, synovial fluid, and urine and may be useful markers of disease progression. The ability to detect biomarkers of cartilage degradation in body fluids may enable clinicians to diagnose sub-clinical OA as well as determining the course of disease progression. New biomarkers that indicate early responses of the joint cartilage to degeneration will be useful in detecting early, pre-radiographic changes. Systems biology is increasingly applied in basic cartilage biology and OA research. Proteomic techniques have the potential to improve our understanding of OA physiopathology and its underlying mechanisms. Proteomics can also facilitate the discovery of disease-specific biomarkers and help identify new therapeutic targets. Proteomic studies of cartilage and other joint tissues may be particularly relevant in diagnostic orthopedics and therapeutic research. This perspective article discusses the relevance and potential of proteomics for studying age-related musculoskeletal diseases such as $\mathrm{OA}$ and reviews the contributions of key investigators in the field.

Keywords: musculoskeletal aging, proteomics, osteoarthritis

\section{THE GLOBAL CHALLENGE OF MUSCULOSKELETAL DISEASES CHARACTERIZED AND EXACERBATED BY AGING}

The incidence of age-related diseases is rising, seriously affecting the health of millions of people around the world. According to the United Nations $(\mathrm{UN})^{1}$ and the World Health Organization $(\mathrm{WHO})^{2}$ musculoskeletal, rheumatic, and arthritic conditions are leading causes of morbidity and disability throughout the world, giving rise to enormous healthcare expenditures and loss of work (Woolf and Pfleger, 2003; source: http://www.arthritis.org/) $)^{3,4}$. Many types of rheumatic diseases and arthritic conditions are essentially age-related "inflammatory" disorders where the inflammation facilitates disease progression. The term "arthritis" characterizes a group of conditions involving inflammatory damage to synovial joints (Di Paola and Cuzzocrea, 2008). Arthritis literally means inflammation (itis) of the joints (arthr). It involves pain, redness, heat, swelling, and other harmful effects of inflammation within the joint. There are over 200 different forms of arthritis. However, the most common and important form of arthritis is

\footnotetext{
${ }^{1}$ http://www.un.org/

${ }^{2}$ http://www.who.int/en/

${ }^{3}$ http://www.who.int/healthinfo/statistics/bod_osteoarthritis.pdf

${ }^{4}$ http://whqlibdoc.who.int/bulletin/2003/Vol81-No9/bulletin_2003_81(9)_630.pdf
}

osteoarthritis (OA), also known as osteoarthrosis or degenerative joint disease (DJD). OA is the most prevalent of the chronic diseases affecting the elderly (Aigner et al., 2004). The majority of the population over 65 years of age demonstrate radiographic evidence of $\mathrm{OA}$ in at least one joint. Although $\mathrm{OA}$ is rare in people under 40, it becomes much more common with age. More than 20 million Americans are estimated to have $\mathrm{OA}^{5}$. A 2005 study in the USA estimated that OA is one of the top five causes of disability amongst non-hospitalized adults [source: Center for Disease Control $\left.\left(\mathrm{CDC}^{6}\right), \mathrm{USA}\right]$. In 2006 it was estimated that around 35 million to 40 million Europeans suffer from OA and nearly $25 \%$ of people aged 60 and above suffer from OA induced disability. It is also anticipated that by the year 2030,20\% of adults will have developed OA in Western Europe and North America. Therefore, $\mathrm{OA}$ is expected to place a heavy economic burden on healthcare systems and community services throughout the world. The risk factors for $\mathrm{OA}$ are well known and include age, overweight/obesity, underlying metabolic or endocrine disease, genetics, and joint trauma (Lotz and Kraus, 2010). With increasing life expectancy, growth in the elderly population and an alarming escalation of

\footnotetext{
${ }^{5}$ http://www.niams.nih.gov/

${ }^{6} \mathrm{http}: / /$ www.cdc.gov/
} 
chronic, inflammatory, and age-related conditions (such as OA), there is increased demand for new treatments and preventative approaches.

\section{ARTICULAR CARTILAGE STRUCTURE AND FUNCTION}

Articular cartilage is the main tissue involved in OA. It is a mechanically unique and resilient connective tissue responsible for load-bearing and low-friction movement in the synovial joints of all vertebrates (Buckwalter et al., 2005). Cartilage is avascular and as a consequence it has a very limited capacity for intrinsic repair (Brittberg, 1999; Tew et al., 2001). It highly prone to structural degradation making it particularly difficult to restore once it is damaged or lost. The extracellular matrix (ECM) of cartilage gives the tissue resilience and elasticity. The ECM consists of three classes of molecules: collagens, aggregating proteoglycans, and non-collagenous proteins. Type II, IX, and XI collagens form a fibrillar framework of macromolecules that give the tissue form, tensile stiffness, and mechanical strength (Buckwalter and Mankin, 1998b; Eyre, 2004). Large aggregating proteoglycans (predominantly aggrecan) allow cartilage to swell and resist compressive forces (Hardingham and Fosang, 1992; Kuettner, 1992). Small proteoglycans including decorin, biglycan, and fibromodulin, bind to other matrix macromolecules and help to stabilize the ECM. Other collagenous and non-collagenous macromolecules present within the ECM perform a variety of structural and informational roles, facilitate cell-cell and cell-matrix interactions, and bind growth factors (Hardingham and Fosang, 1992; Feng et al., 2006). The chondrocyte is the only cell type present in articular cartilage (Archer and Francis-West, 2003). During embryonic development chondrocytes synthesize a cartilaginous template for endochondral ossification and skeletal development and in postnatal life they maintain the ECM by regulating the turnover of matrix components in response to biomechanical, biochemical, and endocrine signals (Goldring and Marcu, 2009). Chondrocytes actively synthesize new ECM components as well as the proteolytic enzymes such as matrix metalloproteinases (MMPs), a disintegrin, and metalloproteinase (ADAMs) and a disintegrin and metalloproteinase with thrombospondin motifs (ADAMTSs) are responsible for tissue remodeling during development. These enzymes are also involved in the catabolic breakdown of cartilage in OA (Aigner et al., 2006).

\section{CARTILAGE DEGRADATION IN OSTEOARTHRITIS}

Osteoarthritis is a degenerative disease that involves joint inflammation, bone remodeling, and catabolic destruction of the articular cartilage component (Goldring and Goldring, 2007; Samuels et al., 2008). In OA there is an imbalance between the synthesis and degradation of ECM macromolecules (Felson, 2004). This can be due to increased enzymatic activity of MMPs (Okada et al., 1992), and pro-inflammatory mediators such as cytokines (Goldring and Goldring, 2004), prostaglandins, and nitric oxide (Goldring and Berenbaum, 2004), coupled with the reduced anabolic capacity of chondrocytes (Aigner et al., 1997) and the tissue's inherently poor reparative capacity due to its avascular nature (Archer and Francis-West, 2003). Consequently OA is characterized by the loss of structural constituents from the ECM. The degradation and release of proteins and glycoproteins from cartilage in OA can vary according to the stage of the disease process. For example, elevated serum cartilage oligomeric matrix protein (COMP) is correlated with the presence of OA and disease severity (Clark et al., 1999).

\section{AGING AND OSTEOARTHRITIS}

Aging is a major contributor to musculoskeletal degeneration and the development of OA (Hamerman, 1998; Lotz and Carames, 2011). Age-related changes in articular cartilage contribute to the development and progression of OA. Although the degeneration of articular cartilage is not simply the result of aging and mechanical wear, aging nevertheless modifies the articular joint including cartilage, subchondral bone, muscle, soft tissues, synovial membrane, and synovial fluid (Buckwalter and Mankin, 1998a; Hamerman, 1998). Although older age is the greatest risk factor for OA, OA is not an inevitable consequence of growing old (Shane Anderson and Loeser, 2010). The mechanisms for the link between aging and OA are incompletely understood. Cell stress and oxidative damage contribute to chronic inflammation that promotes age-related diseases. In OA this results in senescence-associated secretory phenotype, which has many of the characteristics of an osteoarthritic chondrocyte in terms of the cytokines, chemokines, and proteases produced (Loeser, 2011).

\section{BIOMARKERS OF OSTEOARTHRITIS}

A major focus of clinical research in recent years has been the identification of new disease markers that can facilitate early diagnosis and optimize individualized treatments. Such markers can also facilitate the drug discovery process by reducing the high levels of attrition in clinical trials. A biomarker is classically defined as a biochemical entity that is used to measure the progress of a disease or the effects of treatment on clinical outcome. Biochemical markers can be measured in blood, serum, and urine or a variety of other body fluids and tissues. The National Cancer Institute $(\mathrm{NCI})^{7}$ defines a biomarker as "a biological molecule found in blood, other body fluids, or tissues that is a sign of a normal or abnormal process, or of a condition or disease," and the terms "molecular markers" or "signature molecules" have also been used to describe such markers. The term biomarker is all encompassing and can include proteins, protein fragments, metabolites, carbohydrates, nucleic acids (RNA and DNA), cellular features, and images.

Osteoarthritis is unambiguously diagnosed when it is "detected" by the best available test. Thus far the best test for this purpose has been radiography, the so-called "gold-standard." This process also requires clinical signs in the patient, which often occur well into the progression of the disease. However, there is often early, pre-clinical evidence of disease provided by various biomarkers, which if detected, may facilitate earlier diagnosis and treatment. Such an approach is particularly pertinent in the case of OA, a disease often characterized by a prolonged pre-clinical "molecular" phase, a "pre-radiographic" phase, and a "recalcitrant radiographic" phase by which time there are structural changes to joints along with pain and loss of function. Biomarkers have the potential to provide an early warning of joint degeneration

${ }^{7}$ http://www.cancer.gov/ 
which could prompt earlier, more targeted treatment to prevent the tissue destruction that results in the characteristic chronic disability associated with OA. In this context, biomarkers could make a significant contribution to the early diagnosis of OA, as well as informing key aspects of disease prognosis, monitoring, and therapy.

The National Institute of Arthritis and Musculoskeletal and Skin Diseases (NIAMS) established the Osteoarthritis Biomarkers Network $^{8}$ to develop and validate standardized, sensitive biomarker assays in blood and urine to facilitate the diagnosis of the pre-radiologic stage of OA in humans and in animal models. Such markers can help us understand the biological processes involved in disease progression and allow us to monitor the effects of surgical or pharmacological treatment, thus accelerating the pace of drug discovery. Such biomarkers could also potentially be used to identify patients at increased risk of developing OA. Existing biomarkers of OA have major limitations: they do not "flag" the pre-radiographic phase of the disease; they are not specific for the various stages of OA, and in some cases, may not even be specific for OA.

Considering these challenges, the Osteoarthritis Research Society International (OARSI) ${ }^{9}$ and the US Food and Drug Administration (FDA $)^{10}$ have recently established a new OA biomarkers working group, which has proposed the division of potential markers into two major groups: the so-called soluble or "wet" biomarkers, which typically reflect a modulation in an endogenous substance in body fluids such blood, serum, plasma, urine, or synovial fluid); and the "dry" biomarkers, which usually consist of visual analog scales, performed tasks, or images of joints (Kraus et al., 2011).

Therefore, the ability to detect biomarkers of cartilage degradation and/or inflammation in biological samples, such as serum, urine, or synovial fluid, may enable clinicians to diagnose subclinical OA as well as determining the disease stage in both human and companion animals. Identifying these biomarkers will also aid drug discovery and drug safety/efficacy monitoring in patients and in animal models. Using combinations of biomarkers may be more effective in achieving these goals, thus having a panel of biomarkers will help researchers and the pharmaceutical industry to monitor disease progression as well as to assess responses to treatment in experimental models of OA (Rousseau and Delmas, 2007; Williams, 2009).

\section{SYSTEMS BIOLOGY AND PROTEOMIC APPROACHES FOR THE DISCOVERY OF OSTEOARTHRITIS BIOMARKERS}

Systems biology is increasingly applied in orthopedics and rheumatology to cartilage and synovium in arthritis. These techniques include genomics, transcriptomics, proteomics, metabolomics, glycomics, and bioinformatics and can be applied to the study of cartilage, synovium, synovial fluid, and even blood (serum) or urine from OA patients. Proteomics involves the application of specialized analytical techniques that allow the evaluation of the protein composition of tissues, cells, and culture supernatants.

\footnotetext{
${ }^{8}$ http://www.nih.gov/niams/

${ }^{9} \mathrm{http}: / /$ www.oarsi.org/

${ }^{10} \mathrm{http}: / /$ www.fda.gov/
}

Proteomics is being increasingly applied in basic cartilage biology (Polacek et al., 2010) and OA research (Ruiz-Romero et al., 2010). Characterization of cell lysates from isolated chondrocytes has yielded valuable information regarding the intracellular proteins of the chondrocyte proteome, and paved the way for future studies on cartilage pathologies such as OA (Ruiz-Romero et al., 2005; Ruiz-Romero and Blanco, 2010). Studies of soluble proteins in cartilage tissue from OA patients has increased the knowledge of the proteins contained within the ECM of diseased versus normal tissue (Wu et al., 2007). A number of papers have reported on proteins secreted from the cartilage ECM in response to pathological insults such as interleukin (IL)- $1 \alpha$ and all-trans-retinoic acid (Wilson et al., 2008a,b; Ruiz-Romero and Blanco, 2010), IL-1 $\beta$ and TNF- $\alpha$ (Cillero-Pastor et al., 2010) and mechanical compression (Stevens et al., 2008; Zhang and Wang, 2009; Li et al., 2010). Identifying proteins released from cartilage has the potential to give an indication of disease biomarkers likely to be present in the synovial fluid or blood of patients in the early stages of OA.

\section{RELEVANCE OF BIOMARKERS AND PROTEOMIC TECHNIQUES TO "PHYSIOLOGY AND PATHOPHYSIOLOGY OF MUSCULOSKELETAL AGING"}

Understanding healthy aging is a key research priority, along with a better understanding of the pathophysiology of aging that occurs in a number of age-related diseases, such as arthritis. By gaining a better understanding of healthy musculoskeletal aging we can provide better care and new therapies for common musculoskeletal problems. "Physiology and Pathophysiology of Musculoskeletal Aging" is a Research Topic that is intended to bring together basic researchers and clinicians working in the broad area of musculoskeletal aging. The topic includes mechanisms of healthy aging in tissues of the musculoskeletal system (i.e., skeletal muscle, articular cartilage, subchondral bone, tendon, and ligament).

The discovery and validation for biomarkers of OA has accelerated significantly as our understanding of joint tissue molecules and their complex interactions have increased (Kraus, 2005). One of the main drivers in this context has been the urgent need for improved OA "outcome measures" in clinical trials (Kraus, 2005; Hunter et al., 2010). In particular there is a pressing need for new biomarkers that indicate early responses of the joint cartilage to degeneration that will be useful in detecting early, pre-radiographic changes. Novel markers that characterize the status and prognosis of OA, and that can be used to monitor response to therapy are also required (Mobasheri and Henrotin, 2010). Current "omics-based" research aims to develop an "analytical toolbox" which is hoped will contribute to the clinical development process (Bay-Jensen et al., 2010; Qvist et al., 2010). Combinations of existing biomarkers may improve their prognostic accuracy and help identify at-risk patients (Williams, 2009). The challenge is to use proteomics and other "omics-based" technologies in order to identify sensitive and reliable pre-radiographic biomarkers that can be accurately and reproducibly measured in body fluids. Biomarkers that "flag" early stage OA will be particularly useful in curbing disease progression by identifying patients that would benefit from early therapeutic intervention.

In this Research Topic Gharbi and co-workers (Gharbi et al., 2011) review the applications of proteomic techniques for studying OA. Their aim is to improve our understanding of the 
physiopathology of the disease its underlying mechanisms and to discover disease-specific biomarkers and identify new therapeutic targets. This timely and focused review summarizes the currently available data regarding proteomic techniques and their applications to OA research. The authors discuss technical limitations and solutions to real and practical problems including sample preparation. Although proteomics has many potential applications in this area, there are technical challenges that still remain. This elegant and original article highlights the major issues facing researchers in this area.

\section{REFERENCES}

Aigner, T., Rose, J., Martin, J., and Buckwalter, J. (2004). Aging theories of primary osteoarthritis: from epidemiology to molecular biology. Rejuvenation Res. 7, 134-145.

Aigner, T., Soeder, S., and Haag, J. (2006). IL-1beta and BMPs - interactive players of cartilage matrix degradation and regeneration. Eur. Cell. Mater. 12, 49-56.

Aigner, T., Vornehm, S. I., Zeiler, G., Dudhia, J., von der Mark, K., and Bayliss, M. T. (1997). Suppression of cartilage matrix gene expression in upper zone chondrocytes of osteoarthritic cartilage. Arthritis Rheum. (Munch.) 40, 562-569.

Archer, C. W., and Francis-West, P. (2003). The chondrocyte. Int. J. Biochem. Cell Biol. 35, 401-404.

Bay-Jensen, A. C., Sondergaard, B. C., Christiansen, C., Karsdal, M. A., Madsen, S. H., and Qvist, P. (2010). Biochemical markers of joint tissue turnover. Assay Drug Dev. Technol. 8, 118-124.

Brittberg, M. (1999). Autologous chondrocyte transplantation. Clin. Orthop. Relat. Res. S147-S155.

Buckwalter, J. A., and Mankin, H. J. (1998a). Articular cartilage: degeneration and osteoarthritis, repair, regeneration, and transplantation. Instr. Course Lect. 47, 487-504.

Buckwalter, J. A., and Mankin, H. J. (1998b). Articular cartilage: tissue design and chondrocyte-matrix interactions. Instr. Course Lect. 47, 477-486.

Buckwalter, J. A., Mankin, H. J., and Grodzinsky, A. J. (2005). Articular cartilage and osteoarthritis. Instr. Course Lect. 54, 465-480.

Cillero-Pastor, B., Ruiz-Romero, C., Carames, B., Lopez-Armada, M. J., and Blanco, F. J. (2010). Proteomic analysis by two-dimensional electrophoresis to identify the normal human chondrocyte proteome stimulated by tumor necrosis factor alpha and interleukin-1beta. Arthritis Rheum. (Munch.) 62, 802-814.
Clark, A. G., Jordan, J. M., Vilim, V., Renner, J. B., Dragomir, A. D., Luta, G., and Kraus, V. B. (1999). Serum cartilage oligomeric matrix protein reflects osteoarthritis presence and severity: the Johnston County Osteoarthritis Project. Arthritis Rheum. (Munch.) 42, 2356-2364.

Di Paola, R., and Cuzzocrea, S. (2008). Predictivity and sensitivity of animal models of arthritis. Autoimmun. Rev. 8, 73-75.

Eyre, D. R. (2004). Collagens and cartilage matrix homeostasis. Clin. Orthop. Relat. Res. S118-S122.

Felson, D. T. (2004). An update on the pathogenesis and epidemiology of osteoarthritis. Radiol. Clin. North Am. 42, 1-9.

Feng, H., Danfelter, M., Stromqvist, B., and Heinegard, D. (2006). Extracellular matrix in disc degeneration. $J$. Bone Joint Surg. Am. 88(Suppl. 2), 25-29.

Gharbi, M., Deberg, M., and Henrotin, Y. (2011). Application for proteomic techniques in studying osteoarthritis: a review. Front. Physio. 2:90. doi: 10.3389/fphys.2011.00090.

Goldring, M. B., and Berenbaum, F. (2004). The regulation of chondrocyte function by proinflammatory mediators: prostaglandins and S37-S46.

Goldring, M. B., and Goldring, S. R. (2007). Osteoarthritis. J. Cell. Physiol. 213, 626-634.

Goldring, M. B., and Marcu, K. B. (2009). Cartilage homeostasis in health and rheumatic diseases. Arthritis Res. Ther. 11, 224.

Goldring, S. R., and Goldring, M. B. (2004). The role of cytokines in cartilage matrix degeneration in osteoarthritis. Clin. Orthop. Relat. Res. S27-S36.

Hamerman, D. (1998). Biology of the aging joint. Clin. Geriatr. Med. 14, 417-433.

Hardingham, T. E., and Fosang, A. J. (1992). Proteoglycans: many forms and many functions. FASEB J. 6, 861-870. nitric oxide. Clin. Orthop. Relat. Res.

\section{ACKNOWLEDGMENTS}

The author wishes to acknowledge the collaboration of the WALTHAM Centre for Pet Nutrition and the generous financial support of The Wellcome Trust, the National Centre for the Replacement, Refinement, and Reduction of Animals in Research (NC3Rs; grant number: Mobasheri. A. 28102007), the Biotechnology and Biological Sciences Research Council (BBSRC; grants BBSRC/S/M/2006/13141 and BB/G018030/1) and the Engineering and Physical Sciences Research Council (EPSRC).

Hunter, D. J., Losina, E., Guermazi, A., Burstein, D., Lassere, M. N., and Kraus, V. (2010). A pathway and approach to biomarker validation and qualification for osteoarthritis clinical trials. Curr. Drug Targets 11, 536-545.

Kraus, V. B. (2005). Biomarkers in osteoarthritis. Curr. Opin. Rheumatol. 17, 641-646.

Kraus, V. B., Burnett, B., Coindreau, J., Cottrell, S., Eyre, D., Gendreau, M., Gardiner, J., Garnero, P., Hardin, J., Henrotin, Y., Heinegård, D., Ko, A., Lohmander, L. S., Matthews, G., Menetski, J., Moskowitz, R., Persiani, S., Poole, A. R., Rousseau, J. C., Todman, M., and OARSI FDA Osteoarthritis Biomarkers Working Group. (2011). Application of biomarkers in the development of drugs intended for the treatment of osteoarthritis. Osteoarthr. Cartil. 19, 515-542.

Kuettner, K. E. (1992). Biochemistry of articular cartilage in health and disease. Clin. Biochem. 25, 155-163.

Li, H., Yang, H. S., Wu, T. J., Zhang, X. Y., Jiang, W. H., Ma, Q. L., Chen, Y. X., Xu, Y., Li, S., and Hua, Z. C. (2010). Proteomic analysis of early-response to mechanical stress in neonatal rat mandibular condylar chondrocytes. J. Cell. Physiol. 223, 610-622.

Loeser, R. F. (2011). Aging and osteoarthritis. Curr. Opin. Rheumatol. 23, 492-496.

Lotz, M. K., and Carames, B. (2011) Autophagy and cartilage homeostasis mechanisms in joint health, aging and OA. Nat. Rev. Rheumatol. 7, 579-587.

Lotz, M. K., and Kraus, V. B. (2010) New developments in osteoarthritis. Posttraumatic osteoarthritis: pathogenesis and pharmacological treatment options. Arthritis Res. Ther. 12, 211.

Mobasheri, A., and Henrotin, Y. (2010). Identification, validation and qualification of biomarkers for osteoarthritis in humans and companion animals: mission for the next decade. Vet. J. 185 , 95-97.

Okada, Y., Shinmei, M., Tanaka, O., Naka, K., Kimura, A., Nakanishi, I., Bayliss, M. T., Iwata, K. and Nagase, H. (1992). Localization of matrix metalloproteinase 3 (stromelysin) in osteoarthritic cartilage and synovium. Lab. Invest. 66, 680-690.

Polacek, M., Bruun, J. A., Johansen, O., and Martinez, I. (2010). Differences in the secretome of cartilage explants and cultured chondrocytes unveiled by SILAC technology. J. Orthop. Res. 28, 1040-1049.

Qvist, P., Christiansen, C., Karsdal, M. A., Madsen, S. H., Sondergaard, B. C., and Bay-Jensen, A. C. (2010). Application of biochemical markers in development of drugs for treatment of osteoarthritis. Biomarkers 15, 1-19.

Rousseau, J. C., and Delmas, P. D. (2007). Biological markers in osteoarthritis. Nat. Clin. Pract. Rheumatol. 3, 346-356.

Ruiz-Romero, C., and Blanco, F. J. (2010). Proteomics role in the search for improved diagnosis, prognosis and treatment of osteoarthritis. Osteoarthr. Cartil. 18, 500-509.

Ruiz-Romero, C., Calamia, V., Carreira, V., Mateos, J., Fernandez, P., and Blanco, F. J. (2010). Strategies to optimize two-dimensional gel electrophoresis analysis of the human joint proteome. Talanta 80 , 1552-1560.

Ruiz-Romero, C., Lopez-Armada, M. J., and Blanco, F. J. (2005). Proteomic characterization of human normal articular chondrocytes: a novel tool for the study of osteoarthritis and other rheumatic diseases. Proteomics 5, 3048-3059.

Samuels, J., Krasnokutsky, S., and Abramson, S. B. (2008). Osteoarthritis: a tale of three tissues. Bull. NYU Hosp. Jt. Dis. 66, 244-250.

Shane Anderson, A., and Loeser, R. F. (2010). Why is osteoarthritis an agerelated disease? Best Pract. Res. Clin. Rheumatol. 24, 15-26. 
Stevens, A. L., Wishnok, J. S., Chai, D. H., Grodzinsky, A. J., and Tannenbaum, S. R. (2008). A sodium dodecyl sulfate-polyacrylamide gel electrophoresis-liquid chromatography tandem mass spectrometry analysis of bovine cartilage tissue response to mechanical compression injury and the inflammatory cytokines tumor necrosis factor alpha and interleukin1beta. Arthritis Rheum. (Munch.) 58, 489-500.

Tew, S., Redman, S., Kwan, A., Walker, E., Khan, I., Dowthwaite, G., Thomson, B., and Archer, C. W. (2001). Differences in repair responses between immature and mature cartilage. Clin. Orthop. Relat. Res. S142-S152.
Williams, F. M. (2009). Biomarkers: in combination they may do better. Arthritis Res. Ther. 11, 130.

Wilson, R., Belluoccio, D., Little, C. B., Fosang, A. J., and Bateman, J. F. (2008a). Proteomic characterization of mouse cartilage degradation in vitro. Arthritis Rheum. (Munch.) 58, 3120-3131.

Wilson, R., Belluoccio, D., Little, C. B., Fosang, A. J., and Bateman, J. F. (2008b). Proteomic characterization of mouse cartilage degradation in vitro. Arthritis Rheum. (Munch.) 58, 3120-3131.

Woolf, A. D., and Pfleger, B. (2003). Burden of major musculoskeletal conditions. Bull. World Health Organ. 81, 646-656.
Wu, J., Liu, W., Bemis, A., Wang E., Qiu, Y., Morris, E. A., Flannery, C. R., and Yang, Z. (2007) Comparative proteomic characterization of articular cartilage tissue from normal donors and patients with osteoarthritis. Arthritis Rheum. (Munch.) 56, 3675-3684.

Zhang, W. B., and Wang, L. (2009). Label-free quantitative proteome analysis of skeletal tissues under mechanical load. J. Cell. Biochem. $108,600-611$.

Conflict of Interest Statement: The author declares that the research was conducted in the absence of any commercial or financial relationships that could be construed as a potential conflict of interest.
Received: 02 December 2011; accepted: 05 December 2011; published online: 23 December 2011.

Citation: Mobasheri A (2011) Applications of proteomics to osteoarthritis, a musculoskeletal disease characterized by aging. Front. Physio. 2:108. doi 10.3389/fphys.2011.00108

This article was submitted to Frontiers in Striated Muscle Physiology, a specialty of Frontiers in Physiology.

Copyright (c) 2011 Mobasheri. This is an open-access article distributed under the terms of the Creative Commons Attribution Non Commercial License, which permits non-commercial use, distribution, and reproduction in other forums, provided the original authors and source are credited. 